Article

\title{
Study on Reduced Cost of Non-Salient Machine System Using MTPA Angle Pre-Compensation Method Based on EEMF Sensorless Control
}

\author{
Kyoung Jin Joo ${ }^{1}$ (D) , Joon Sung Park ${ }^{2}$ (D) and Ju Lee ${ }^{1, *}$ \\ 1 Department of Electrical Engineering, Hanyang University, Seoul 04763, Korea; kj8532@hanyang.ac.kr \\ 2 Intelligent Mechatronics Research Center, Korea Electronics Technology Institute, Gyeonggi-do 13509, Korea; \\ parkjs@keti.re.kr \\ * Correspondence: julee@hanyang.ac.kr; Tel.: +82-2-2220-4349
}

Received: 31 March 2018; Accepted: 31 May 2018; Published: 2 June 2018

\begin{abstract}
When a consumer chooses a product, one of the most important considerations is price. Therefore, cost reduction is the most important factor when manufacturing a product. This paper has applied the sensorless method to remove the position sensor in an interior permanent magnet synchronous motor (IPMSM) system and examined the way to reduce the use of the trigonometric function in order to lower the dependency on a high-end CPU. A sensorless method has been studied to use an extended electromotive force (EEMF) that can increase the degree of freedom of the motor in the IPMSM system. In addition, an observer is applied to the estimated position to stabilize the system. Furthermore, many trigonometric functions are used for driving the IPMSM. Since this trigonometric function requires decimal point operations, that requires a lot of computation time and a high-performance CPU. Therefore, this paper proposes a new method to pre-compensate the maximum torque per ampere (MTPA) angle as a way to reduce the use of the trigonometric functions which makes a high-spec CPU unnecessary. Simulations and experiments are conducted to verify the proposed control algorithm.
\end{abstract}

Keywords: maximum torque per ampere (MTPA); current angle pre-compensation; extended electromotive force (EEMF); sensorless control; motor drive topology

\section{Introduction}

In recent years, high-efficiency products have been preferred due to concerns over high oil prices and energy resources. In particular, there is an increasing demand for higher efficiency of high-output household appliances such as robotic vacuum cleaners, fans, air conditioners, refrigerators and washing machines. The same concern applies to servo drive motors and industrial robots for factory automation systems [1,2].

Permanent Magnet (PM) machines have higher power density, higher power factor and higher efficiency per unit volume than induction motor, and have the advantage in terms of size because there is no heat due to copper loss. Even PM motors are easier to control. In particular, IPMSM can utilize electromagnetic torque $\left(T_{e}\right)$ as well as reluctance torque to output higher torque. Further, since the IPMSM can be designed to have a smaller air gap than surface permanent magnet synchronous motor (SPMSM), the magnetic flux density is also higher. Therefore, IPMSM is attracting attention as the most widely used induction motor replacement motor in industry [2-8].

In descending order the cost proportion of each part of an IPMSM system is: (1) electric machine; (2) power switching devices; (3) position sensors, and (4) digital processor (CPU). The electric machine and the power switching devices are necessary for system operation. Therefore, this paper concentrates 
on efficient cost saving methods for the position sensor and CPU that represent a large proportion of the total cost excluding the electric machine and power switching devices. In a large-capacity motor system, the price of the position sensor or the CPU is not a large portion because the cost of the system itself is high. However, in the case of $4 \mathrm{~kW}$ capacity systems, the cost can be reduced by up to $35 \%$. Therefore, when this method is applied instead of the method that was previously used in the industry, more cost-effective results can be obtained for low-capacity systems.

Constant torque control is required to operate the IPMSM with high efficiency. In order to constantly control the torque, it is necessary to precisely know the rotor position information. For this purpose, a position sensor such as an encoder or resolver is required. However, using the sensor has the disadvantage of increasing the system cost. Therefore, in this paper, a sensorless control method which can estimate the rotor position without sensor is studied to reduce system costs [9-17].

The sensorless method is largely divided into a method using back electromotive force (BEMF) [10] and a method using the inherent characteristics of a motor [11,12]. The method using BEMF is disadvantageous in that it is difficult to generate electromotive force in the low speed region, and the method using the motor parameters has a disadvantage in that it is difficult to accurately estimate due to the fluctuation of the motor parameters in the high output area. Therefore, in this paper, the sensorless control algorithm using EEMF based on BEMF is introduced to avoid these disadvantages. This sensorless method is suitable for IPMSM, because it can estimate the rotor position by introducing the extended EMF concept to the difference between inductance and flux linkage $[9,14]$. However, a high performance and high-speed CPU is required to implement the complex algorithms used for sensorless control. Furthermore, a high-performance CPU capable of decimal point operation is required to the calculation of many trigonometric functions necessary to operate IPMSM, but the high-end CPU also increases the price of the system. Therefore, this paper proposes the MTPA angle pre-compensation method to reduce the computation of the trigonometric functions which occupy a large amount of CPU computation time [18].

In the IPMSM, the MTPA angle, $\beta$, for the maximum-efficiency operating point is non-zero and there exist a non-linear characteristic value due to the inductance difference of the $d q$-axes [3-8]. The $d$-axis reference current is non-zero and has a negative value. This leads to a complicated electromagnetic-torque equation and a trigonometric function calculation. Accordingly, this paper proposes a strategy that can control the IPMSM like the SPMSM by controlling the non-linear characteristic of $\beta$ in advance. Therefore, if the $d$-axis rotor current of the IPMSM can be driven to zero by pre-compensating $\beta$, large torque per volume can be produced and a linear control performance for speed-to-torque conversion over the entire operating range can be realized. To validate this assumption, the strategy is simulated using MATLAB/Simulink (R2018a, The MathWorks, Inc, Natick, MA, USA) and tested with a $4 \mathrm{~kW}$ IPMSM.

\section{Conventional Control Equations}

\subsection{Voltage Equation}

The stator voltage equation of the 3-phase stationary reference-frame for space vector control of the IPMSM can be expressed as:

$$
v_{a b c s}=R_{s} i_{a b c s}+\frac{d \lambda_{a b c s}}{d t}
$$

The voltage in Equation (1) is expressed by a complex time-varying differential equation, except when the motor is at a standstill. Therefore, to more easily interpret such a complex time-varying differential equation, the 3-phase stator flux $\lambda_{a b c s}$ is subjected to the following procedure to convert into $d q$-axes rotating as the electrical angular velocity $\omega_{e}$ of the rotor. The system performance of IPMSM is improved by applying vector control to independently control $d q$-axes for fast response characteristics: 


$$
\begin{gathered}
\lambda_{a b c s}=L_{s} i_{a b c s}+\lambda_{f} \\
T\left(\theta_{e}\right) \lambda_{a b c s}=T\left(\theta_{e}\right) L_{s} i_{a b c s}+T\left(\theta_{e}\right) \lambda_{f} \\
\lambda_{d q r}=T\left(\theta_{e}\right) L_{s}\left(T\left(\theta_{e}\right)^{-1} i_{d q r}\right) i_{d q r}+T\left(\theta_{e}\right) \lambda_{f} \\
T\left(\theta_{e}\right)=\frac{2}{3}\left[\begin{array}{ll}
\cos \theta_{e} & \cos \left(\theta_{e}-\frac{2 \pi}{3}\right) \\
-\sin \theta_{e} & -\cos \left(\theta_{e}-\frac{4 \pi}{3}\right) \\
-\sin \left(\theta_{e}-\frac{2 \pi}{3}\right) & -\sin \left(\theta_{e}-\frac{4 \pi}{3}\right)
\end{array}\right]
\end{gathered}
$$

The voltage equation in the 2-phase rotor coordinate system is expressed as follows by applying the magnetic flux $\lambda_{d q r}$ summarized above:

$$
\left[\begin{array}{c}
v_{d r} \\
v_{q r}
\end{array}\right]=\left[\begin{array}{ll}
R_{s}+p L_{d r} & -\omega_{e} L_{q r} \\
\omega_{e} L_{d r} & R_{s}+p L_{q r}
\end{array}\right]\left[\begin{array}{l}
i_{d r} \\
i_{q r}
\end{array}\right]+\left[\begin{array}{l}
0 \\
\omega_{e} K_{E}
\end{array}\right]
$$

$L_{d r}$ and $L_{q r}$ can be expressed as:

$$
\begin{aligned}
& L_{d r}=l_{a}+\frac{3}{2}\left(L_{a}-L_{a s}\right) \\
& L_{q r}=l_{a}+\frac{3}{2}\left(L_{a}+L_{a s}\right)
\end{aligned}
$$

From Equation (2) to Equation (5), it can be seen that the flux and voltage equations for driving the IPMSM requires the position information $\theta_{e}$ of the rotor. Thus, the position sensor is applied so that precise voltage control can be performed through the accurate position of the rotor. However, the application of the sensor not only increases the system cost, but also lengthens the axial length of the motor, and also restricts installation environment and requires periodic maintenance. In addition, since the noise may be mixed in the signal depending on the environment, the reliability of the system is degraded. Therefore, various sensorless control methods for removing such position sensors have been studied, and sensorless control algorithms can be largely divided as follows: (1) signal injection method; (2) estimation method of rotor magnetic flux or BEMF of stator; (3) application of observer, and (4) method of using motor parameters. Among these various methods, the rotor position estimation method using EEMF which extended concept of BEMF was applied in this paper. Figure 1 shows the general configuration of IPMSM motor control.

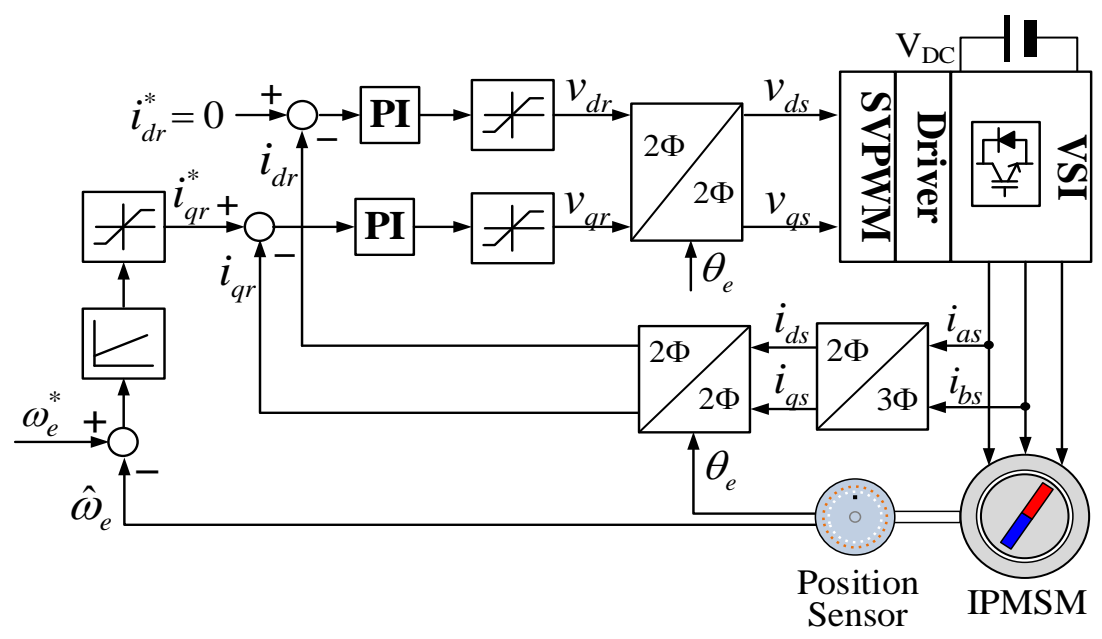

Figure 1. Block diagram of general control for interior permanent magnet synchronous motor (IPMSM). 


\subsection{MTPA Control}

In the case of IPMSM, unlike the SPMSM, electromagnetic torque is generated by the sum of the permanent magnet torque component generated by the interaction of the magnetic flux and the output current and the reluctance torque component generated by the difference between the $d$-axis and the $q$-axis inductances, as shown in the following torque Equation (6). Using this reluctance torque component, a slightly larger torque than the SPMSM can be obtained. To generate this torque, a $d$-axis current is required and $d$-axis current should be negative because $L_{q r}$ is greater than $L_{d r}$. If the flux by the permanent magnet is constant and the inductance difference does not change, the torque can be instantaneously controlled by controlling the magnetic flux axis current and the torque axis current, respectively:

$$
\begin{aligned}
& T_{e}=\frac{3}{2} \frac{P}{2}\left\{\lambda_{f} i_{q r}+\left(L_{d r}-L_{q r}\right) i_{d r} i_{q r}\right\} \\
& =\frac{3}{2} \frac{P}{2}\left\{\lambda_{f} i_{s} \cos \beta+\left(L_{d r}-L_{q r}\right) i_{s} \cos \beta i_{s} \sin \beta\right\} \\
& =\frac{3}{2} \frac{P}{2}\left\{\lambda_{f} i_{s} \cos \beta+\frac{1}{2}\left(L_{d r}-L_{q r}\right) i_{s}^{2} \sin 2 \beta\right\}
\end{aligned}
$$

The combinations of $i_{d r}$ and $i_{q r}$ capable of generating $T_{e}$, are myriad, as shown in Figure 2. However, the minimum current combination (i.e., the maximum torque output per unit current) that can minimize the copper loss while generating the same electromagnetic torque output is unique. The line connecting these $d q$ current pairs is called the MTPA curve. The operation along the MTPA curve is referred to as MTPA control, and the angle of the curve is referred to as current angle or MTPA angle. MTPA control in IPMSM system is very efficient and important.

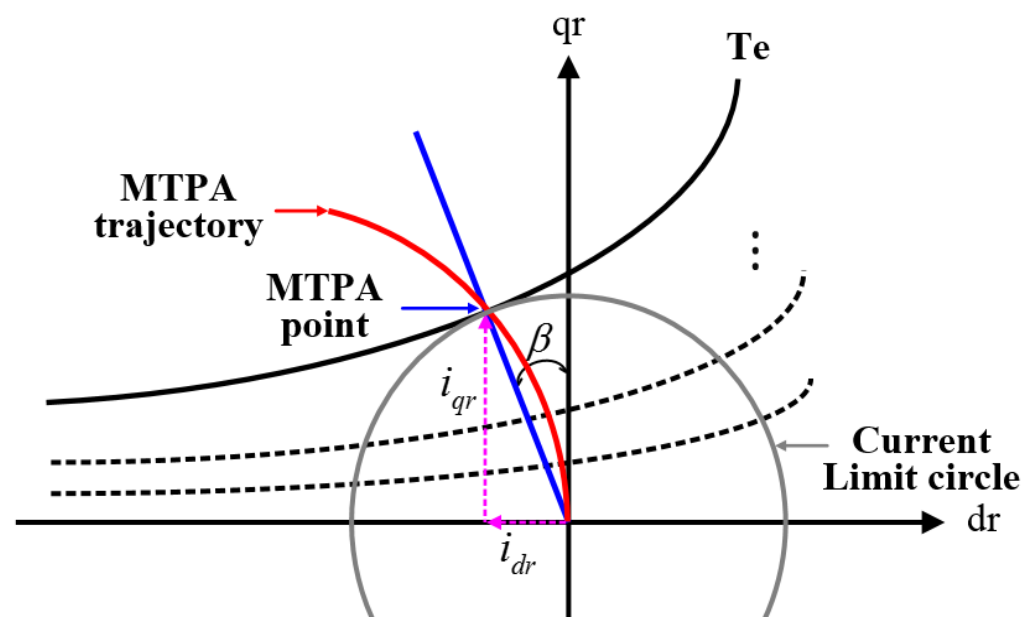

Figure 2. Maximum torque per ampere (MTPA) curve and current relationship diagram.

From the torque Equation (6), $\beta$ can be obtained at current limit condition as follows:

$$
\beta=\sin ^{-1}\left\{\frac{-\lambda_{f}+\sqrt{\lambda_{f}^{2}+8\left(L_{d r}-L_{q r}\right)^{2} i_{s}^{2}}}{4\left(L_{d r}-L_{q r}\right) i_{s}}\right\}
$$

From this, the optimal $d q$-axes instantaneous current commend can be obtained as follows:

$$
\begin{aligned}
& i_{d r}=i_{s} \cos \beta \\
& i_{q r}=i_{s} \sin \beta
\end{aligned}
$$


The $d$-axis inductance for the $d$-axis current fluctuation in the IPMSM is almost constant. However, the variation of the $q$-axis inductance with respect to the $q$-axis current fluctuation is very large, because the $q$-axis in which the magnetic circuit is composed of iron is largely influenced by the magnetic saturation. In addition, due to saturation, the current fluctuations of different axes are affected, and the magnitude of the flux also varies with the operating temperature. Therefore, it is necessary to consider the variation of the motor parameters for accurate MTPA control. In other words, accurate MTPA control is difficult without information about the exact parameters.

Also, for MTPA control, trigonometric functions, which consume much computation time of CPU, are frequently used. Therefore, this paper is proposed a new torque control algorithm of the IPMSM that can be simply replaced with the MTPA control part, which is not accurate due to the variation of the motor parameters during operation and allocates a lot of computation time.

\section{EEMF Sensorless Algorithm}

The voltage Equation (4) in the 2-phase rotating coordinate frame is re-expressed as the voltage Equation (9) in the 2-phase stationary coordinate frame to include information about the rotor position. When the permanent magnet of the IPMSM is rotating, a magnetic flux of the rotor is being induced in the stator winding, and BEMF is being generated. From the induced voltage, information on the rotor position can be estimated. To simplify the voltage Equation (9), Equation (10) can be rewritten by achieving a symmetric form of the impedance matrix. The second term on the right side of Equation $(10)$ is referred to as the extended $\operatorname{EMF}\left(E_{e x}\right)$ :

$$
\begin{gathered}
{\left[\begin{array}{l}
v_{d s} \\
v_{q s}
\end{array}\right]=\left[\begin{array}{ll}
R_{s}+p L_{d s} & -\omega_{e} L_{q s} \\
\omega_{e} L_{d s} & R_{s}+p L_{q s}
\end{array}\right]\left[\begin{array}{l}
i_{d s} \\
i_{q s}
\end{array}\right]+\omega_{e} K_{E}\left[\begin{array}{l}
-\sin \theta_{e} \\
\cos \theta_{e}
\end{array}\right]} \\
{\left[\begin{array}{c}
v_{d s} \\
v_{q s}
\end{array}\right]=\left[\begin{array}{ll}
R_{s}+p L_{d s} & \omega_{e}\left(L_{d s}-L_{q s}\right) \\
-\omega_{e}\left(L_{d s}-L_{q s}\right) & R_{s}+p L_{d s}
\end{array}\right]\left[\begin{array}{l}
i_{d s} \\
i_{q s}
\end{array}\right]+\left[\left(L_{d s}-L_{q s}\right)\left(\omega_{e} i_{d s}-p i_{q s}\right)+\omega_{e} K_{E}\right]\left[\begin{array}{l}
-\sin \theta_{e} \\
\cos \theta_{e}
\end{array}\right]}
\end{gathered}
$$

$E_{e x}$ can be rearranged as shown in Equation (11). If the inductance of the flux axis equals the inductance of the torque axis ( $L_{d s}=L_{q s}$ ), Equation (11a) can be applied (as in the case of SPMSM). Also, when the flux linkage $K_{E}$ is zero (as in the case of the synchronous reluctance motor), Equation (11b) can be applied. Thus, the Equation (11) with several degrees of freedom is applicable to any motor. The sensorless EEMF method has the advantage that $E_{e x}$ can be estimated even at low speed region when the $q$-axis current is fluctuating slightly. Therefore, in this study, the sensorless EEMF method is applied:

$$
\begin{gathered}
{\left[\begin{array}{l}
e_{d s} \\
e_{q s}
\end{array}\right]=\left[\left(L_{d s}-L_{q s}\right)\left(\omega_{e} i_{d s}-p i_{q s}\right)+\omega_{e} K_{E}\right]\left[\begin{array}{l}
-\sin \theta_{e} \\
\cos \theta_{e}
\end{array}\right]} \\
{\left[\begin{array}{l}
e_{d s} \\
e_{q s}
\end{array}\right]=\omega_{e} K_{E}\left[\begin{array}{l}
-\sin \theta_{e} \\
\cos \theta_{e}
\end{array}\right]} \\
{\left[\begin{array}{l}
e_{d s} \\
e_{q s}
\end{array}\right]=\left[\left(L_{d s}-L_{q s}\right)\left(\omega_{e} i_{d s}-p i_{q s}\right)\right]\left[\begin{array}{l}
-\sin \theta_{e} \\
\cos \theta_{e}
\end{array}\right]}
\end{gathered}
$$

The coordinate system of estimated rotor position can be defined with $\gamma \delta$-axes. The position error $\widetilde{\theta}_{e}$ is defined as the difference between the actual position and the estimated position $\left(\widetilde{\theta}_{e}=\theta_{e}-\hat{\theta}_{e}\right)$. The relationship between the $d q r$ - and $\gamma \delta$-axes is shown in Figure 3 below. The sign "^" represents the estimated value. 


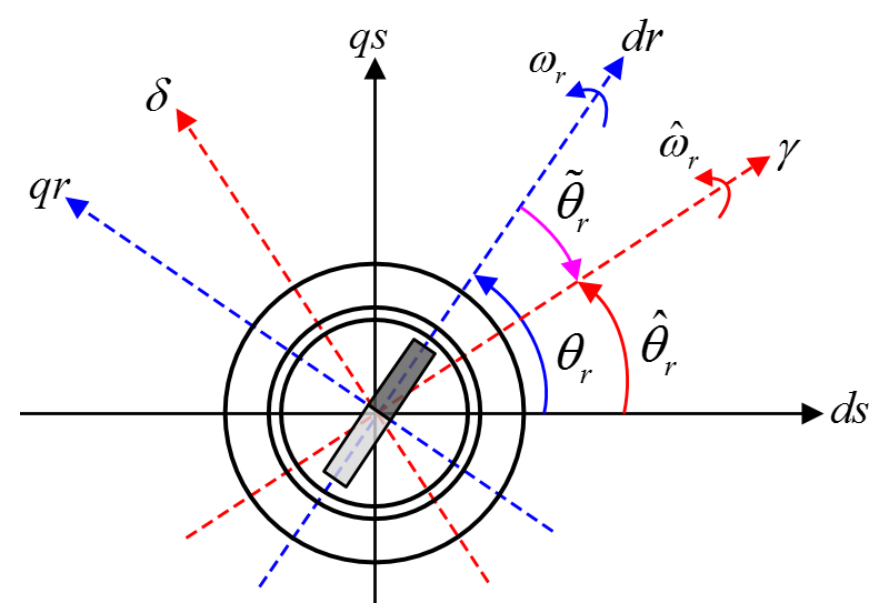

Figure 3. Relationship between rotor positions for extended electromotive force (EEMF) sensorless method.

The EEMF terms of the $\gamma \delta$-axes contain the position estimation error information, which can be obtained using an observer. To eliminate the decoupling term $v_{\delta_{-} f f}$, it is forward compensated and can be expressed as shown in Figure 4. The parameter $g_{o b}$ represents an observer gain and $v_{\gamma}, e_{\gamma}$ and $i_{\gamma}$ are $\gamma$-axis voltage, EMF, and current, respectively.

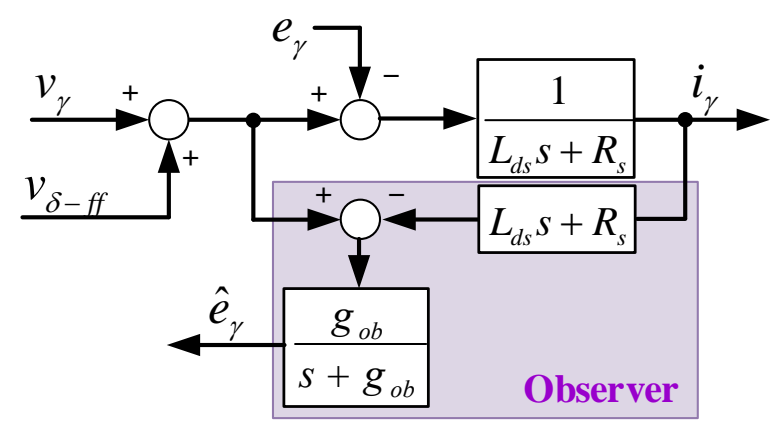

Figure 4. Block diagram of observer to estimate EEMF.

The EEMF equation applied to the observer can be expressed with Equation (12). And assuming that the velocity estimation is reasonable $\left(\hat{\omega}_{e} \simeq \omega_{e}\right)$, the final Equation (13) can be obtained:

$$
\begin{gathered}
{\left[\begin{array}{l}
e_{d s} \\
e_{q s}
\end{array}\right]=E_{e x}\left[\begin{array}{l}
-\sin \theta_{e} \\
\cos \theta_{e}
\end{array}\right]+\left(\hat{\omega}_{e}-\omega_{e}\right) L_{d s}\left[\begin{array}{l}
-i_{\gamma} \\
i_{\delta}
\end{array}\right]} \\
{\left[\begin{array}{l}
\hat{e}_{d s} \\
\hat{e}_{q s}
\end{array}\right]=E_{e x}\left[\begin{array}{l}
-\sin \hat{\theta}_{e} \\
\cos \hat{\theta}_{e}
\end{array}\right]}
\end{gathered}
$$

Therefore, the estimated position of the rotor can be obtained simply by using the EEMF sensorless method and the observer.

\section{Proposed MTPA Angle Compensation Method}

MTPA control is essential for high-efficiency operation of IPMSM. However, since negative $d$-axis current command is generated for MTPA, the current angle $\beta$ has a non-zero value. Furthermore, due to the characteristic of $\beta$, the motor control has nonlinear characteristics and the dependence on magnetic saturation and motor parameters is increased. Therefore, this paper proposes a new method 
for pre-compensation of $\beta$ to eliminate the nonlinear operation characteristics of IPMSM, simplify the computation of algorithm, and reduce the reliance on trigonometric functions. The following Equation (14) represents the $d$-axis current command for MTPA control.

$$
i_{d s}=\frac{-\lambda_{f}+\sqrt{\lambda_{f}^{2}+8\left(L_{d s}-L_{q s}\right)^{2} i_{s}^{2}}}{4\left(L_{d s}-L_{q s}\right)}
$$

Figure 5a shows a general IPMSM vector diagram for controlling a $d q$-axes current pair for MTPA control. General vector control performs two transformations. First, the current vector of the 2-phase stationary reference frame is projected onto the 2-phase rotation reference frame. Second, the current vector of the 2-phase rotation reference frame is projected in the MTPA coordinate system by $\beta$. In this case, the introduction of eight trigonometric functions is essential for the two coordinate transformations. Figure $5 \mathrm{~b}$ suggests a strategy to simplify the double conversion process of Figure $5 \mathrm{a}$. The transformation in Figure $5 \mathrm{~b}$ further rotates MTPA angle $\beta$ to estimated position by the sensorless algorithm, which matches the phase of $q$-axis current with the output phase current. When this method is used, the command of the output phase current appears all over the $q$-axis, and the $d$-axis current does not participate in the distortion of the output current as the $d$-axis current converges to zero. Therefore, the IPMSM controls the $d$-axis current to 0 like the SPMSM, while the controller becomes simple and the MTPA control disappears. Consequently, the IPMSM becomes independent of the influence of the motor parameters and has a linear characteristic.

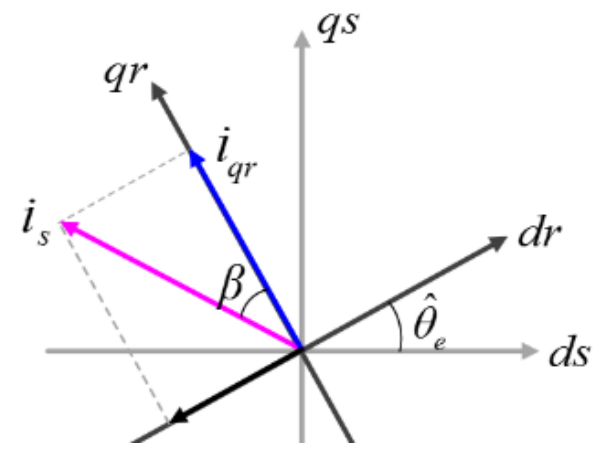

(a)

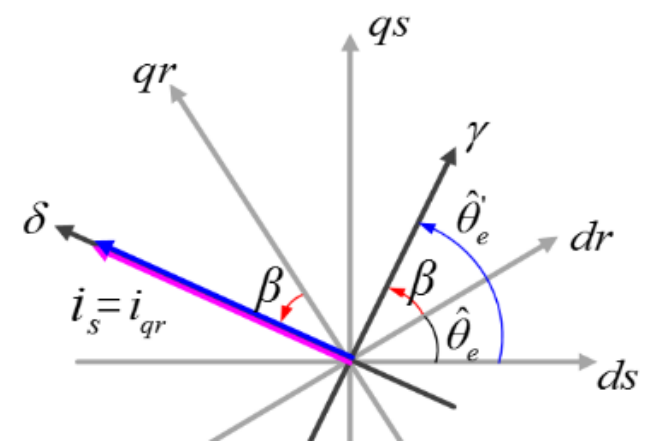

(b)

Figure 5. Comparison of general and proposed current vector diagrams (a) General current vector diagram; (b) advanced current vector diagram.

To prove this assumption, the general coordinate transformation in Figure 5a (transformation from the stationary coordinate system to the rotation coordinates and the MTPA transformation) and the coordinate transformation of the proposed MTPA pre-compensation method in Figure $5 \mathrm{~b}$ are mathematically compared. Figure 5a,b can be expressed as Equations (15) and (16), respectively. Obviously, the results of both conversions (Equations (15) and (16)) are derived in the same way as Equation (17). Therefore, the above assumptions have proven to be problem-free as formulas.

$$
\begin{gathered}
{\left[\begin{array}{l}
i_{d r}^{\prime} \\
i_{q r}^{\prime}
\end{array}\right]=\left[\begin{array}{ll}
\cos \beta & \sin \beta \\
-\sin \beta & \cos \beta
\end{array}\right]\left[\begin{array}{ll}
\cos \theta_{e} & \sin \theta_{e} \\
-\sin \theta_{e} & \cos \theta_{e}
\end{array}\right]\left[\begin{array}{l}
i_{d r} \\
i_{q r}
\end{array}\right]} \\
{\left[\begin{array}{l}
i_{d r}^{\prime} \\
i_{q r}^{\prime}
\end{array}\right]=\left[\begin{array}{ll}
\cos \left(\beta+\theta_{e}\right) & \sin \left(\beta+\theta_{e}\right) \\
-\sin \left(\beta+\theta_{e}\right) & \cos \left(\beta+\theta_{e}\right)
\end{array}\right]\left[\begin{array}{c}
i_{d r} \\
i_{q r}
\end{array}\right]} \\
{\left[\begin{array}{l}
i_{d r}^{\prime} \\
i_{q r}^{\prime}
\end{array}\right]=\left[\begin{array}{ll}
\cos \theta_{e} \cos \beta-\sin \theta_{e} \sin \beta & \cos \theta_{e} \sin \beta+\sin \theta_{e} \cos \beta \\
-\left(\sin \theta_{e} \cos \beta+\cos \theta_{e} \sin \beta\right) & \cos \theta_{e} \cos \beta-\sin \theta_{e} \sin \beta
\end{array}\right]\left[\begin{array}{c}
i_{d r} \\
i_{q r}
\end{array}\right]}
\end{gathered}
$$


In addition, a phase-locked loop (PLL) system is constructed to obtain a more accurate rotor position from the estimated position error. The position information $\hat{\theta}_{e}^{\prime}$ is a value pre-compensated by adding $\beta$ to $\hat{\theta}_{e}$. The PLL system is configured to estimate the rotor speed by applying a proportional-integral (PI) controller to the error value between the actual position information and the estimated position information. Figure 6 shows the PLL system. And the overall motor speed control block diagram with the sensorless mode proposed in Sections 3 and 4 is shown in Figure 7.

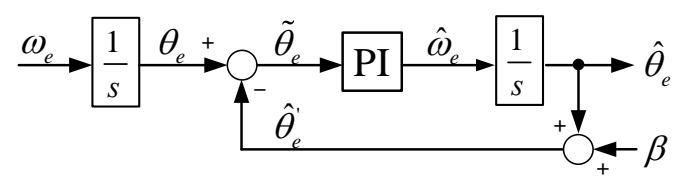

Figure 6. Block diagram of phase-locked loop (PLL) system.

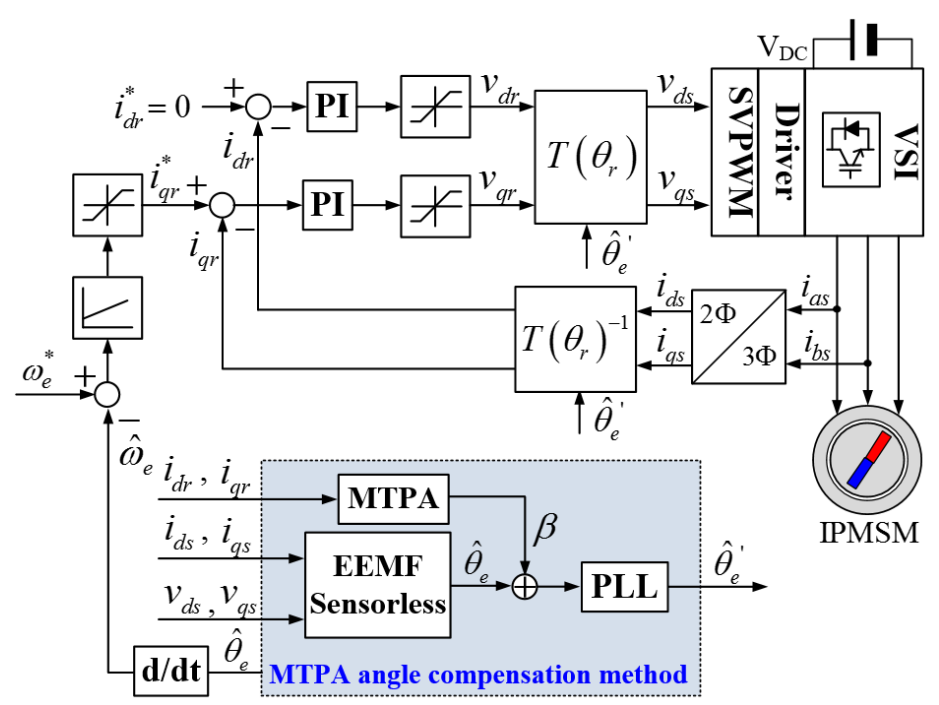

Figure 7. Overall sensorless speed control block diagram with advanced MTPA mechanism for IPMSM.

\section{Simulation}

Simulation with MATLAB/Simulink was conducted to verify the validity of the sensorless control using the EEMF estimation method and the proposed pre-compensation algorithm for the MTPA angle. Table 1 shows detailed parameter specifications of the IPMSM used in the simulation and experiment.

Table 1. Specifications of used IPMSM model.

\begin{tabular}{cc}
\hline Parameters & Values \\
\hline Power $(\mathrm{kW})$ & 4 \\
Poles & 10 \\
Rated speed $(\mathrm{rpm})$ & 3500 \\
Rated current $(\mathrm{A})$ & 11.3 \\
Rated torque $(\mathrm{Nm})$ & 11 \\
$L d(\mathrm{mH})$ & 7.095 \\
$L q(\mathrm{mH})$ & 11.027 \\
$R s(\Omega)$ & 0.33 \\
$\Phi(\mathrm{V} \cdot \mathrm{s})$ & 53.1 \\
\hline
\end{tabular}

$\Phi$ is the phase induced voltage magnitude at $1000 \mathrm{rpm}$. The simulated system consists of a conventional sensored model and two sensorless models. The results of each model are compared in Figures 8 and 9. The simulation is implemented at rated speed and torque conditions. The angle $\beta$ measures $18^{\circ}$. 

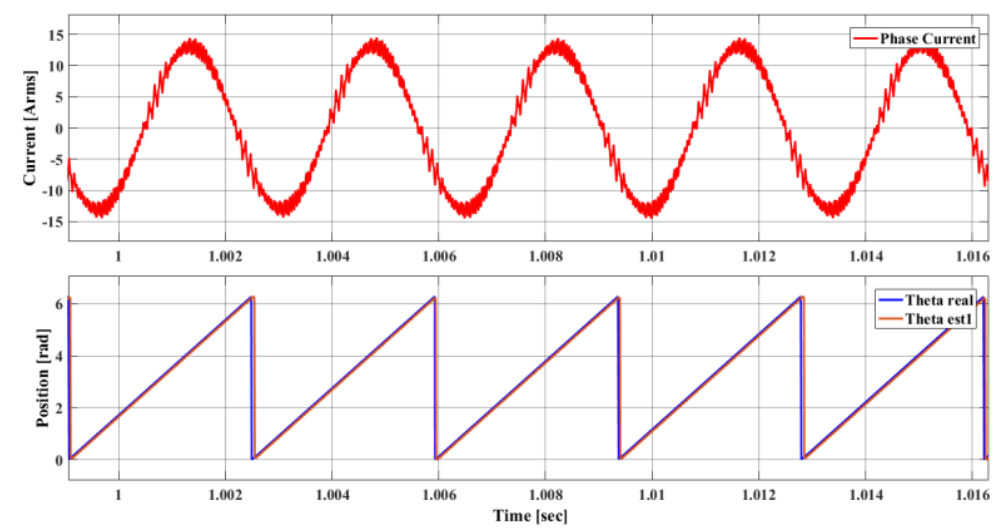

(a)

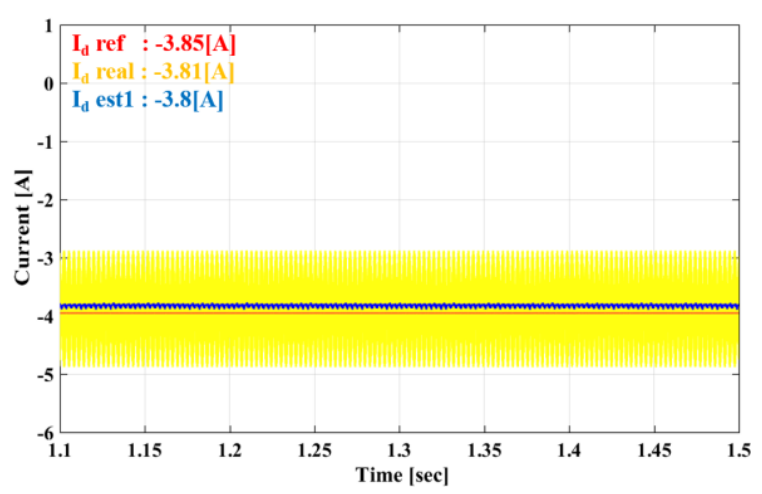

(b)

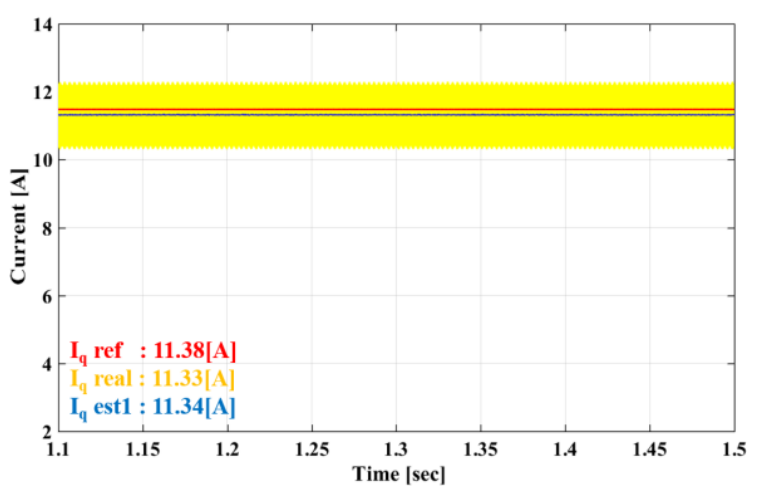

(c)

Figure 8. Simulation results of sensorless EEMF mode at $3500 \mathrm{rpm} / 11 \mathrm{Nm}$ (a) a-phase current and rotor angles $\left(\theta_{e}, \hat{\theta}_{e}\right) ;(\mathbf{b}) d$-axis current comparison; (c) $q$-axis current comparison.

The upper curve in Figure 8a shows the a-phase output current waveform of the IPMSM, and the lower curve represents the real and estimated rotor position comparison waveforms. In Figure 8b,c, the yellow curves are the actual $d q$-axes currents, the blue curves show $d q$-axes the reference currents, and the red curves represent the estimated $d q$-axes currents. Estimation method 1 (labelled est 1 ) in Figure $8 b, c$ is the simulation model with the EEMF sensorless method.

From the simulation results of Figure 8, the estimated position value and the actual position value are almost overlapped (since no delay appears and the sensorless controller operates well). In addition, the estimated $d q$-axes currents reliably follow the reference current values. In Figure 9, estimation method 2 (labelled est 2) represents the algorithm in which the proposed MTPA angle compensation control is further applied to the est 1 method. As shown in Figure 8, the upper part of Figure 9a is the a-phase output current waveform and the lower part is the real and the estimated rotor position comparison waveforms. In contrast to Figure 8, a-phase gap occurs between the actual position and the estimated position, since the MTPA angle $\beta$ has been compensated to the rotor position. In Figure $9 \mathrm{~b}, \mathrm{c}$, the yellow curves represent the actual $d q$-axes currents, the blue curves represent the $d q$-axes reference currents, and the red curves express the estimated $d q$-axes currents with the est 2 method. According to the simulation results in Figure 9, the estimated position accurately tracks the actual position with phase delay of $\beta$. Further, the $d$-axis command current has negative values, whereas it can be confirmed that the $d$-axis current of the est 2 method with the applied MTPA compensation algorithm converges to $0 \mathrm{~A}$. Additionally, it is confirmed that error magnitude of the rotor position and the output current is very small (within $\pm 0.1^{\circ}, \pm 0.1 \mathrm{~A}$ ), when comparing the three schemes, which are sensored model and the sensorless models (est 1, est 2). 

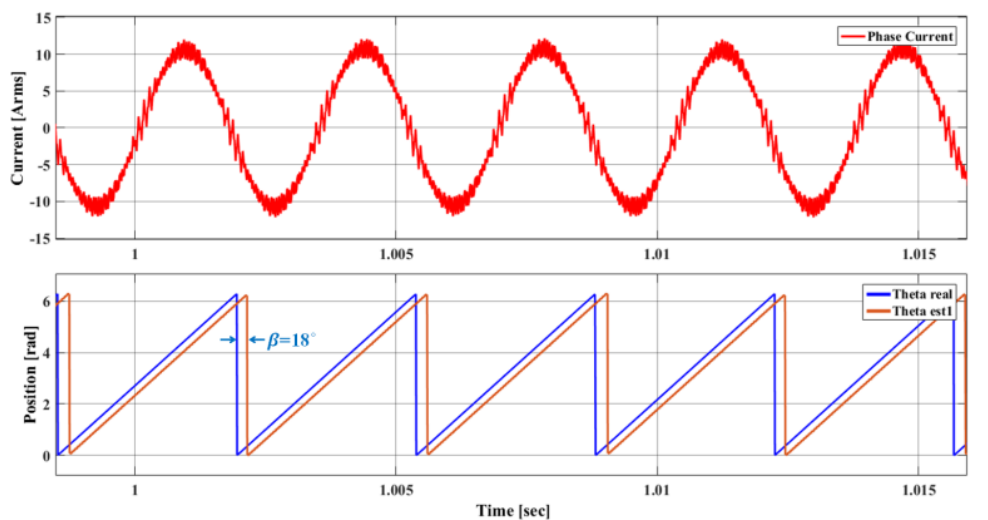

(a)

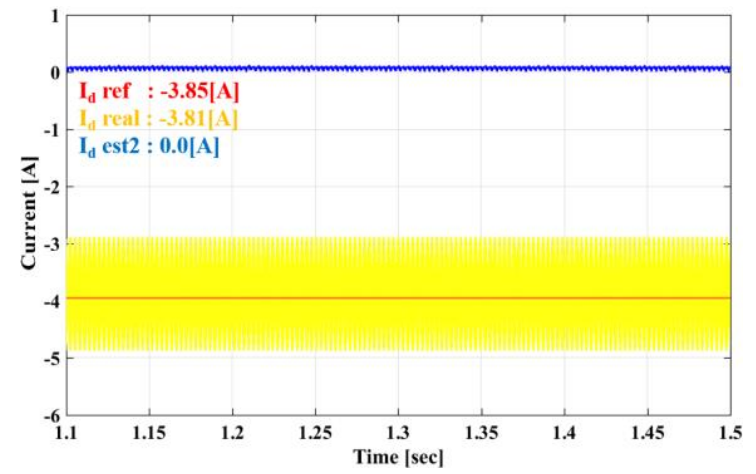

(b)

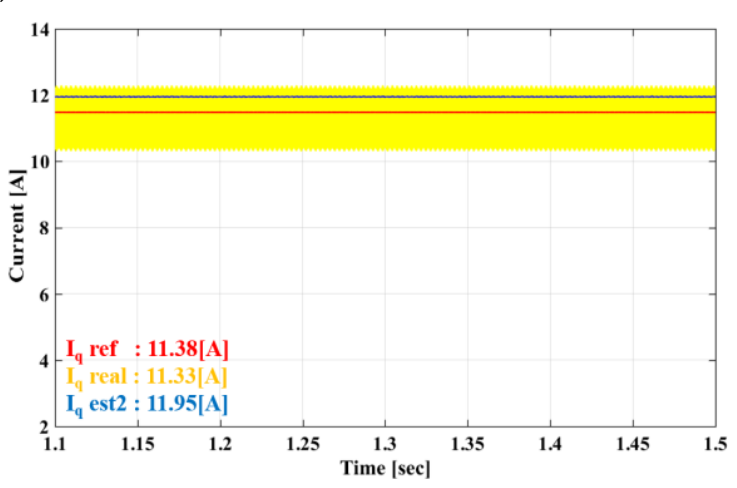

(c)

Figure 9. Simulation results of MTPA angle pre-compensation mode at $3500 \mathrm{rpm} / 11 \mathrm{Nm}$ (a) a-phase current and rotor angles $\left(\theta_{e}, \hat{\theta}_{e}\right)$; (b) $d$-axis current comparison; (c) $q$-axis current comparison.

Finally, Figure 10 shows a comparison of the reference speed, actual speed, and estimated speed on the est 2 model. The estimated speed follows the reference speed reliably and its responsiveness converges rapidly. According to the simulation results, the sensorless method and the proposed MTPA angle-compensation algorithm have proven to be excellent methods. These results will be further confirmed through experiments.

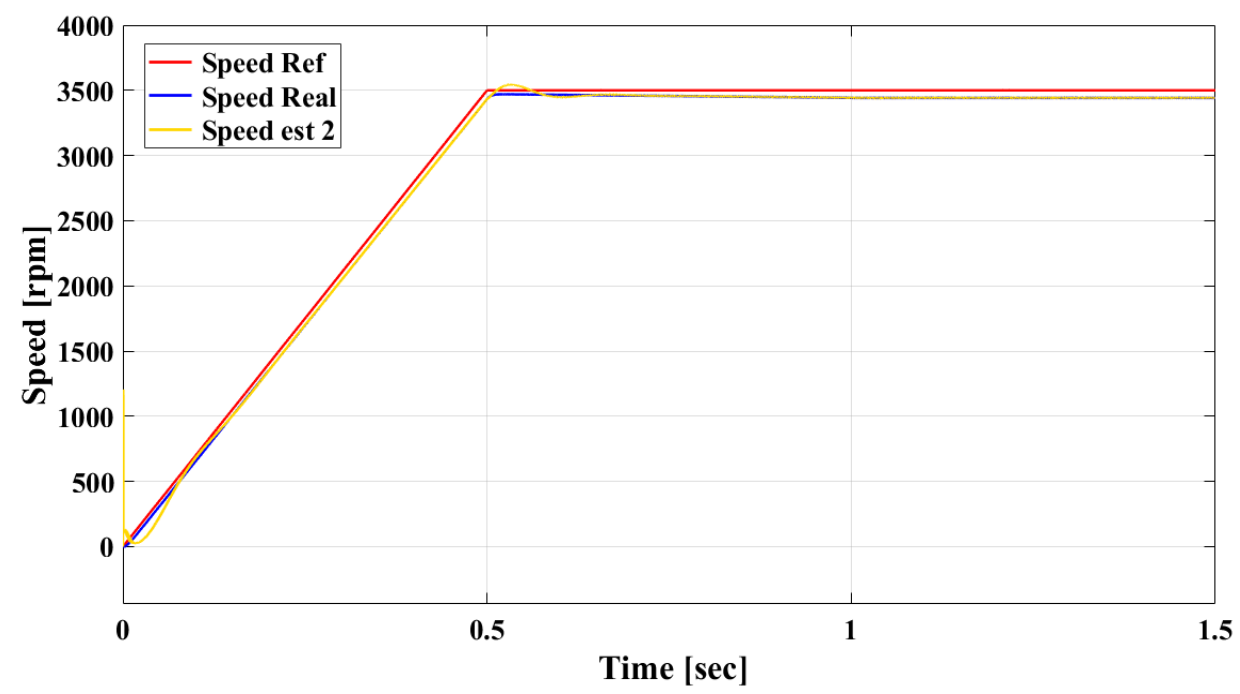

Figure 10. Speed comparison at $3500 \mathrm{rpm} / 11 \mathrm{Nm}$. 


\section{Experimental Results}

The experimental setup and the used motor are shown in Figure 11. The motor is the IPMSM with $4 \mathrm{~kW}, 3500 \mathrm{rpm}$, and 10 poles and it is for an oil pump. The controller was configured using a Texas Instruments' TMS320F28335 (Texas Instruments, Inc, Dallas, TX, USA) unit. The current-control sampling period and pulse width modulation (PWM) switching frequency were set to $20 \mathrm{kHz}$.

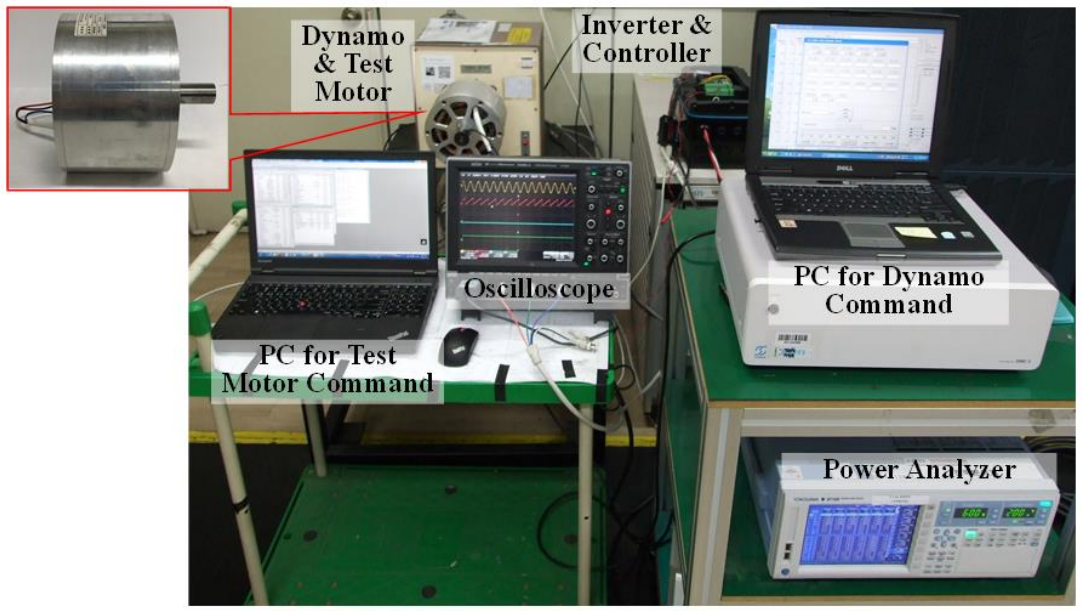

Figure 11. Experimental setup and test motor (4 kW IPMSM).

Figure 12 shows the result applying the EEMF algorithm to the target IPMSM. In Figure 12, the zero point between the output current and the electrical angle of the rotor is slightly different from the simulation due to a delay caused by the differential/integrator of the program and hardware. However, the experimental results are the same as those of the simulation: the $d$-axis current has a negative value of approximately $4 \mathrm{~A}$, and the $q$-axis current measures approximately $11.3 \mathrm{~A}$, as in Figure 8.

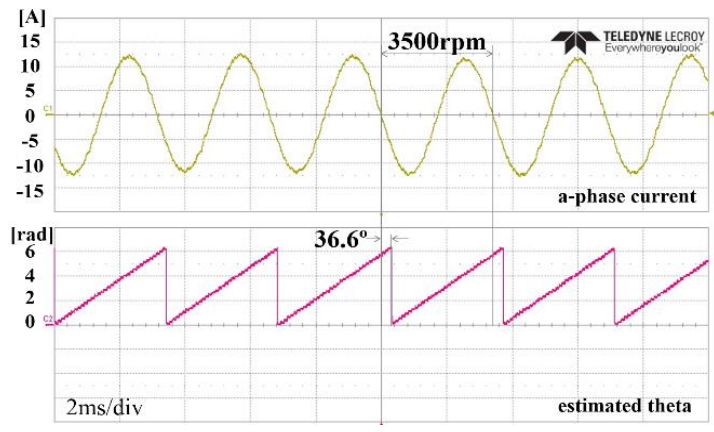

(a)

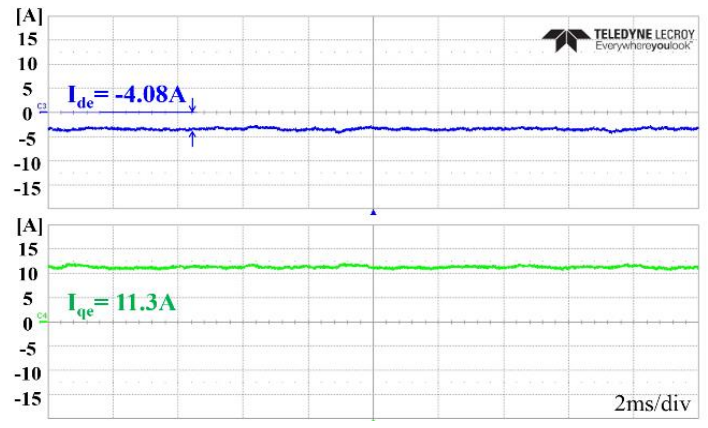

(b)

Figure 12. Experimental results of sensorless EEMF mode at $3500 \mathrm{rpm} / 11 \mathrm{Nm}$. (a) a-phase current and theta; (b) $d q r$-axes currents.

Figure 13 shows the experimental results of the proposed MTPA pre-compensation algorithm. As the results, it can be seen that the $d$-axis current converges to $0 \mathrm{~A}$, all the output phase current appear to the $q$-axis current as shown in Figure 9. Also, the MTPA angle pre-compensation algorithm shows that the MTPA angle $\left(18^{\circ}\right)$ is more delayed than the current phase in the sensorless model. The output currents of Figures 12 and 13 are equally measured as $12 \mathrm{~A}$ within a deviation of $0.01 \mathrm{~A}$. 


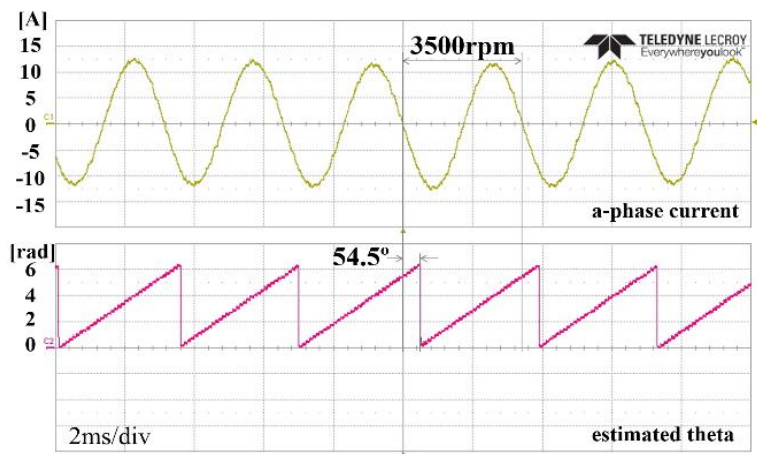

(a)

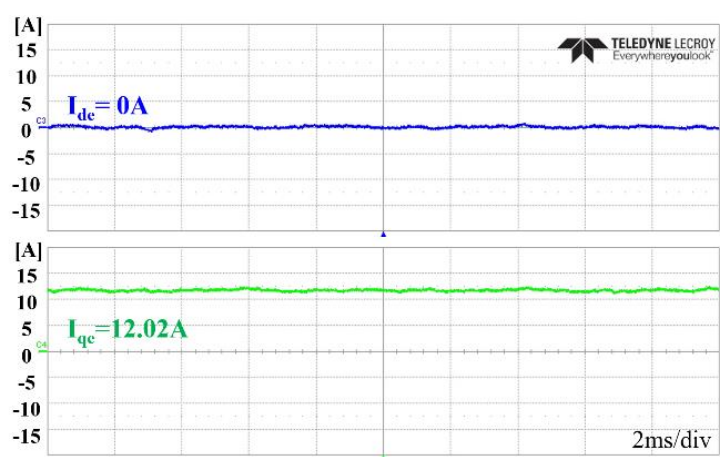

(b)

Figure 13. Experimental results of MTPA angle pre-compensation mode at $3500 \mathrm{rpm} / 11 \mathrm{Nm}$ (a) a-phase current and theta; (b) dqr-axes currents.

\section{Conclusions}

This paper proposes a new method to reduce the cost of industrial motor system by introducing: (1) EEMF sensorless control to remove the position sensor and (2) a MTPA angle pre-compensation method which reduces trigonometric functions to reduce the burden of using a high-performance CPU. Applying this new concept not only removes sensors, but also reduces the trigonometric calculation process, which takes up a lot of computation time in the CPU, resulting in a cost savings of at least $10 \%$ to up to $35 \%$ in the overall IPMSM motor system (including controller, inverter and position sensor). In addition, the nonlinear characteristics by the MTPA control which is a disadvantage of IPMSM are improved, and excellent control performance can be implemented even under rated conditions. As a result, this advanced algorithm maintains the same level of performance while reducing the cost of the system, and makes the motor control more stable and linear. However, the operating range of the oil pump motor used in this paper does not have a low speed section, thus the stability in the extremely low speed range has not been verified. Due to the nature of the EEMF algorithm, stability at low speeds is guaranteed to some extent, but additional control stability must be verified even at extremely low speeds and zero speeds. Therefore, future studies will add adaptive mechanism tests to verify the operating characteristics when applying the proposed algorithm at the extremely low speed and zero speed. Furthermore, the verification of the motor linearity in the field weakening region will proceed.

Author Contributions: K.J.J. developed the proposed modeling approach and validated it through simulation. J.S.P. conceived the main concept of the modelling approach. J.S.P. and K.J.J. performed the experiments and analyzed the data. And J.L. guided and revised the manuscript. All authors contributed to the writing of the manuscript.

Acknowledgments: This work was supported in part by the Human Resources Program in Energy Technology of the Korea Institute of Energy Technology Evaluation and Planning under Grant 20174030201750, and in part by the National Research Foundation of Korea grant funded by the Korea government (Ministry of Science, ICT \& Future Planning) under Grant 2016R1A2A1A05005392.

Conflicts of Interest: The authors declare no conflict of interest. 


\section{Nomenclature}

$\begin{array}{lll}\text { Symbol } & \text { Signification } & \text { Symbol } \\ v_{a b c s} & \text { 3-phase stationary frame voltage } & \lambda_{f} \\ i_{a b c s} & \text { 3-phase stationary frame current } & \lambda_{d q r} \\ i_{d q r} & \text { 2-phase rotating frame current } & T_{e} \\ R_{s} & \text { stator resistance } & E_{e x} \\ L_{s} & \text { stator inductance } & \theta_{e} \\ L_{d q s} & \text { 2-phase stationary frame inductances } & \hat{\theta} \\ L_{d q r} & \text { 2-phase rotating frame inductances } & \widetilde{\theta}_{e} \\ l_{a} & \text { equivalent leakage inductance } & K_{E} \\ L_{a} & \text { average value of the equivalent effective } & T\left(\theta_{e}\right) \\ & \text { inductance } & \\ L_{a s} & \text { amplitude of the effective inductance } & T\left(\theta_{e}\right)^{-1} \\ \omega_{e} & \text { electrical angular velocity } & \hat{\omega}_{e}\end{array}$

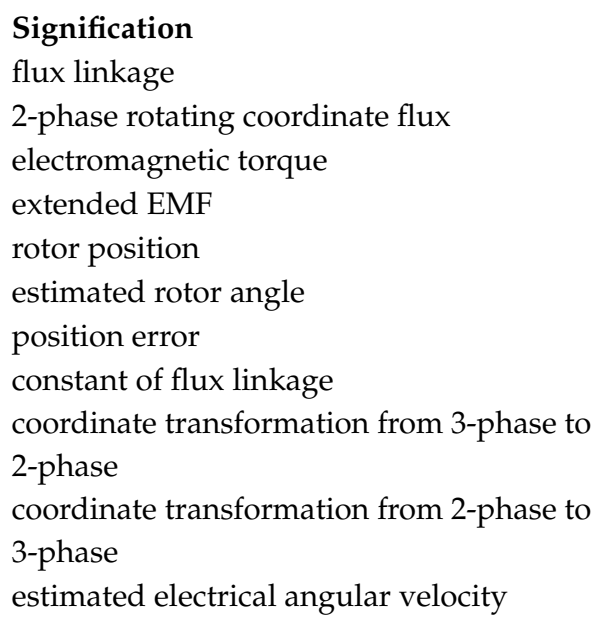

\section{References}

1. Ha, K.; Lee, C.; Kim, J.; Krishnan, R.; Oh, S.G. Design and Development of Low-Cost and High-Efficiency Variable-Speed Drive System With Switched Reluctance Motor. IEEE Trans. Ind. Appl. 2007, 43, 703-713. [CrossRef]

2. Krishnan, R. Permanent Magnet Synchronous and Brushless DC Motor Drives; CRC Press: Boca Raton, FL, USA, 2010.

3. Inoue, T.; Inoue, Y.; Morimoto, S.; Sanada, M. Maximum Torque Per Ampere Control of a Direct Torque-Controlled PMSM in a Stator Flux Linkage Synchronous Frame. IEEE Trans. Ind. Appl. 2016, 52, 2360-2367. [CrossRef]

4. Sun, T.; Wang, J.; Chen, X. Maximum Torque Per Ampere (MTPA) Control for Interior Permanent Magnet Synchronous Machine Drives Based on Virtual Signal Injection. IEEE Trans. Power Electr. 2015, 30, 5036-5045. [CrossRef]

5. Wallmark, O.; Lundberg, S.; Bongiorno, M. Input Admittance Expressions for Field-Oriented Controlled Salient PMSM Drives. IEEE Trans. Power Electr. 2012, 27, 1514-1520. [CrossRef]

6. Pairo, H.; Shoulaie, A. Effective and Simplified Method in Maximum Efficiency Control of Interior Permanent Magnet Synchronous Motors. IET Electr. Power Appl. 2017, 11, 447-459. [CrossRef]

7. Schoonhoven, G.; Uddin, M.N. MTPA- and FW-Based Robust Nonlinear Speed Control of IPMSM Drive Using Lyapunov Stability Criterion. IEEE Trans. Ind. Appl. 2016, 52, 4365-4374. [CrossRef]

8. Liu, Q.; Hameyer, K. High-Performance Adaptive Torque Control for an IPMSM With Real-Time MTPA Operation. IEEE Trans. Energy Conv. 2017, 32, 571-581. [CrossRef]

9. Morimoto, S.; Kawamoto, K.; Sanada, M.; Takeda, Y. Sensorless Control Strategy for Salient-Pole PMSM Based on Extended Emf in Rotating Reference Frame. IEEE Trans. Ind. Appl. 2002, 38, 1054-1061. [CrossRef]

10. Genduso, F.; Miceli, R.; Rando, C.; Galluzzo, G.R. Back EMF Sensorless-Control Algorithm for High-Dynamic Performance PMSM. IEEE Trans. Ind. Electr. 2010, 57, 2092-2100. [CrossRef]

11. Ichikawa, S.; Tomita, M.; Doki, S.; Okuma, S. Sensorless Control of Permanent-Magnet Synchronous Motors Using Online Parameter Identification Based on System Identification Theory. IEEE Trans. Ind. Electr. 2006, 53, 363-372. [CrossRef]

12. Mizutani, R.; Takeshita, T.; Matsui, N. Current Model-Based Sensorless Drives of Salient-Pole PMSM at Low Speed and Standstill. IEEE Trans. Ind. Appl. 1998, 34, 841-846. [CrossRef]

13. Wang, G.; Yang, R.; Xu, D. DSP-Based Control of Sensorless IPMSM Drives for Wide-Speed-Range Operation. IEEE Trans. Ind. Electr. 2013, 60, 720-727. [CrossRef]

14. Kim, H.; Harke, M.C.; Lorenz, R.D. Sensorless Control of Interior Permanent-Magnet Machine Drives with Zero-Phase Lag Position Estimation. IEEE Trans. Ind. Appl. 2003, 39, 1726-1733.

15. Chan, T.F.; Wang, W.; Borsje, P.; Wong, Y.K.; Ho, S.L. Sensorless Permanent-Magnet Synchronous Motor Drive Using a Reduced-Order Rotor Flux Observer. IET Electr. Power Appl. 2008, 2, 88-98. [CrossRef] 
16. Awan, H.A.A.; Tuovinen, T.; Saarakkala, S.E.; Hinkkanen, M. Discrete-Time Observer Design for Sensorless Synchronous Motor Drives. IEEE Trans. Ind. Appl. 2016, 52, 3968-3979. [CrossRef]

17. Davari, S.A.; Khaburi, D.A.; Wang, F.; Kennel, R.M. Using Full Order and Reduced Order Observers for Robust Sensorless Predictive Torque Control of Induction Motors. IEEE Trans. Power Electr. 2012, 27, 3424-3433. [CrossRef]

18. Bear, H.S. Understanding Calculus; 2nd Edition; Wiley-IEEE Press: Hoboken, NJ, USA, 2003.

(C) (1)

(C) 2018 by the authors. Licensee MDPI, Basel, Switzerland. This article is an open access article distributed under the terms and conditions of the Creative Commons Attribution (CC BY) license (http:/ / creativecommons.org/licenses/by/4.0/). 